\title{
Dual-band Substrate Integrated Waveguide Textile Antenna with Integrated Solar Harvester
}

\author{
Sam Lemey, Frederick Declercq and Hendrik Rogier, Senior Member, IEEE
}

\begin{abstract}
A dual-band wearable textile antenna based on substrate integrated waveguide technology is presented for operation in the [2.4-2.4835] GHz Industrial, Scientific and Medical band and the $[2.5-2.69]$ GHz 4G LTE band 7 . The antenna features an integrated flexible solar harvesting system, consisting of a flexible solar cell, a power management system and energy storage. All these components are judiciously positioned on the antenna platform in order not to affect its radiation performance. The measured reflection coefficients and radiation characteristics after bending and deploying the antenna on a human body prove that the antenna is well suited for onbody use. A measured on-body antenna gain and radiation efficiency of $5.0 \mathrm{dBi}$ and $89 \%$ are realized. Measurements in a real-life situation have demonstrated the ability to scavenge a maximum of $53 \mathrm{~mW}$ by means of a single integrated flexible solar cell.
\end{abstract}

Index Terms-Textile antenna, wearable antenna, substrate integrated waveguide (SIW), energy harvesting, energy management, energy storage.

\section{INTRODUCTION}

- mart fabric interactive textile (SFIT) systems play a key role in critical professional applications such as monitoring rescue workers during interventions and patients in hospitals and home-care. Therefore, they set up reliable wireless communication links between the mobile users and the control center. Obviously, end users impose stringent requirements on wearable wireless communication systems, such as stable energy-efficient performance in harsh operating conditions, combined with wearability and comfort. In terms of antenna, a sufficiently broad radiation pattern, a high frontto-back ratio (FTBR) and large radiation efficiency are needed, besides flexibility and breathability. Moreover, to avoid heavy batteries and frequent recharging, low power

This paragraph of the first footnote will contain the date on which you submitted your paper for review.

This research was partially funded by the Inter-University Attraction Poles Program initiated by the Belgian Science Policy Office.

S. Lemey, F. Declercq and H. Rogier are with the Department of Information Technology, IMEC/Ghent University, 9000 Ghent, Belgium (email: sam.lemey@intec.UGent.be). consumption is called for. This makes $2.45 \mathrm{GHz}$ Industrial, Scientific and Medical (ISM) band textile antennas, implementing high data-rate wireless audio/video communication links, ideal for SFIT systems, provided wireless local area network (WLAN) access points are available at the location of operation. In absence of this infrastructure, a 4G Long-Term Evolution (LTE) wireless metropolitan network (WMAN) may serve as an alternative.

While other components miniaturize continuously, the textile antenna's dimensions remain of the order of the wavelength to yield large radiation efficiency in proximity of the human body [1]. Yet, professional garments offer a sufficiently large platform to integrate a textile antenna while active electronic circuitry, such as low-noise amplifier and transceiver, may be directly integrated onto its feed plane [2]. In addition, to extend its autonomy, the system may be powered using energy harvesters [3],[4] by direct integration of solar cells on the antenna patch [5],[6].

Therefore, we present a novel dual-band wearable textile solar antenna for operation in the [2.4-2.4835] GHz ISM band and the 4G LTE band 7 ([2.5 - 2.69] GHz). By implementing a cavity-backed slot antenna in substrate integrated waveguide (SIW) technology, high robustness against bending and stable characteristics in proximity of the human body are achieved. In addition, a flexible solar cell, a power management system (PMS) and micro-energy cell (MEC) are easily integrated without affecting the antenna's radiation performance. Hence, the antenna surface serves simultaneously as efficient wireless communication, energy harvesting and power management platform. To the best of the authors' knowledge, this is the first $2.45 \mathrm{GHz}$ ISM/LTE band 7 dual-band wearable antenna with integrated solar harvester in literature.

Dual-band textile antennas for the bands covered by the proposed antenna have been described in literature before. [7] presents a dual-band $\mathrm{WiFi}$ and 4G LTE textile antenna operating in the [2.4-2.69] $\mathrm{GHz}$ and [5.1-5.875] GHz bands. However, its gain is smaller than $2 \mathrm{dBi}$ in the lower band. The absence of a ground plane between antenna and human body makes the antenna less suitable for on-body use. In contrast, [8] and [9] proposed different textile realizations of cavitybacked antennas based on the SIW technology. Such topologies are very suitable for on-body use [8]-[10]. Yet, both designs are narrow-band due to the excitation of only one resonance frequency, although [11]-[13] outline techniques to enhance the impedance bandwidth. 


\section{DESIGN OF SIW CAVITY-BACKED SLOT ANTENNA}

We first design a wearable textile antenna with a return loss larger than $10 \mathrm{~dB}$ in the $2.45 \mathrm{GHz}$ ISM band ([2.4-2.4835] $\mathrm{GHz}$ ) and in the uplink and downlink of the 4G LTE band 7 ([2.50-2.57] GHz and [2.62-2.69] GHz, respectively).

The geometrical configuration of the proposed SIW cavitybacked slot antenna is shown in Fig. 1. This topology realizes a very high isolation from its surroundings [12] making it suitable for body-worn applications and as platform for the integration of electronic hardware. In addition, an excellent suppression of unwanted surface waves, a large FTBR and a high radiation efficiency $\left(\eta_{r a d}\right)$ is achieved [10] without the need for a large ground plane. SIW technology achieves a simple low-profile and cost-effective single substrate realization by means of tube eyelets, enabling easy routing of the solar cell's DC+ connection without affecting the antenna performance. The bandwidth enhancement technique presented in [13] was applied to realize good bandwidth performance even in the presence of material inhomogeneity, fabrication inaccuracy and frequency de-tuning caused by bending or proximity of the human body.

A rectangular SIW cavity is split into two halves (parts A and B on Fig. 1(a)) by a non-resonant rectangular slot. The dimensions of the slot and both parts of the cavity were carefully chosen to simultaneously excite two hybrid modes, being two different combinations of the $\mathrm{TE}_{110}$ and the $\mathrm{TE}_{120}$ resonances, at resonance frequencies close enough to yield a return loss larger than $10 \mathrm{~dB}$ in the frequency range of interest. The hybrid mode at the lowest resonance frequency is a combination of a strong $\mathrm{TE}_{120}$ and a weak $\mathrm{TE}_{110}$ resonance. The fields in both cavity parts are out of phase, while the dominant field is situated in cavity part A. This results in radiation by the slot. The hybrid mode at the highest resonance frequency is a combination of a strong $\mathrm{TE}_{110}$ and a weak $\mathrm{TE}_{120}$ resonance. The fields in both cavity parts are in phase, but exhibit a large difference in magnitude with the dominant field situated in cavity part B, generating radiation by the slot. A $50 \Omega$ grounded coplanar waveguide (GCPW) feed line is designed in the bottom metal layer (Fig. 1.c). In contrast to [8], impedance matching is realized without extending the GCPW feed line beyond the slot, providing more place to integrate electronic circuitry and improving shielding from the human body. Computer-aided optimization was performed using the transient solver of CST Microwave studio, yielding the antenna dimensions given in Table I.

The proposed antenna is fabricated using pure copper polyester Taffeta, with a surface resistivity $R_{s}=0.18 \Omega / \mathrm{sq}$ [14], as top and bottom layer. A $3.94 \mathrm{~mm}$ thick closed cell expanded rubber protective foam with $\varepsilon_{r}=1.495$ and $\tan \delta$ $=0.016$ at $2.45 \mathrm{GHz}$ serves as substrate [14]. After gluing the two conductive fabrics to the substrate with thermally activated adhesive sheets, the backed cavity is constructed by a row of equally spaced flat flange copper tube eyelets along each side of the cavity.

\section{INTEGRATION OF FLEXIBLE SOLAR ENERGY HARVESTER}

The surface required by the antenna is then reused as a platform for solar energy harvesting and power management.
Therefore, a flexible solar cell is integrated on top of the antenna. A PMS and a MEC are deployed on the antenna feed plane, as shown in Fig. 1.c. Only a single MEC and solar cell are applied here. Yet, depending on the application, more solar cells and energy storage devices may be added to the antenna.
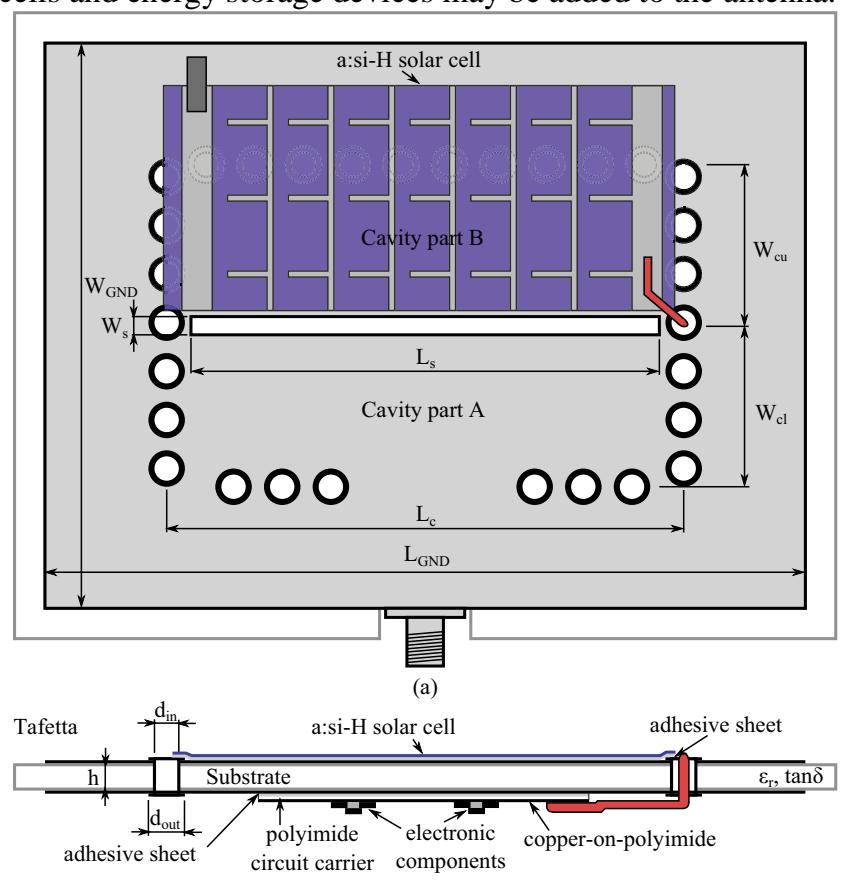

(b)

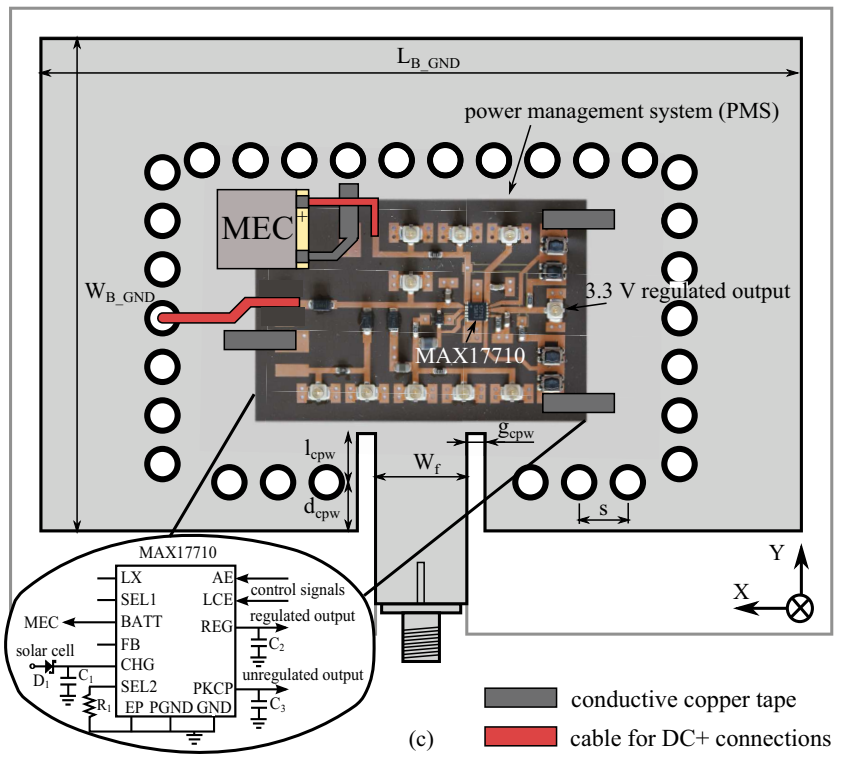

Fig. 1. (a) Top, (b) cross-sectional and (c) bottom view of the proposed SIW cavity-backed slot antenna with integrated energy harvesting hardware. Inset: circuit representation of the PMS ( $D_{1}=$ DFLS130L by Diodes Inc, $C_{1}=$ $\left.0.22 \mu \mathrm{F}, \mathrm{C}_{2}=1 \mu \mathrm{F}, \mathrm{C}_{3}=10 \mu \mathrm{F}, \mathrm{R}_{1}=150 \Omega\right)$.

\section{A. Solar cell integration}

The antenna provides a useful area of $11280 \mathrm{~mm}^{2}$ for solar cell integration, of which $84 \mathrm{~mm}$ x $37 \mathrm{~mm}$ is effectively exploited by one SP4.2-37 ultra-thin and ultra-flexible PowerFilm ${ }^{\circledR}$ thin film a-Si:H solar cell. The solar cell is positioned such that the radiating slot remains uncovered (Fig. 1.a). The DC+ contact wire is routed through one of the tube eyelets to connect with the PMS (Fig. 1). 
TABLE I ANTENNA DIMENSIONS

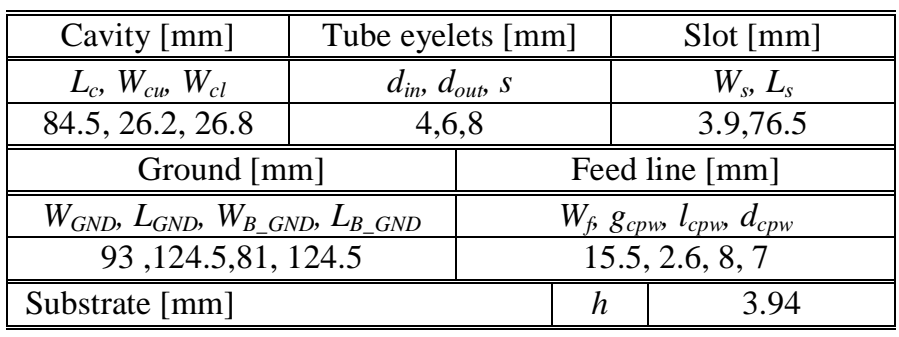

\section{B. Power management circuit integration}

The PMS is built around the MAX17710, a general-purpose energy-harvesting charger and protector IC by Maxim Integrated $^{\mathrm{tm}}$, as depicted in the inset of Fig. 1.c. The PMS controls the charging process of a $170 \mu \mathrm{m}$ thick Thinergy ${ }^{\circledR}$ MEC-225 micro-energy cell and generates a regulated output voltage to adequately power a wireless communication module. In addition, the PMS also protects the MEC against overcharge and overdischarge. The PMS is a linear harvesting system which allows a lower break-even threshold than maximum power point tracking [15]. Hence solar energy harvesting is enabled in lower light intensity situations. The circuit is implemented on a flexible substrate consisting of one polyimide layer and two copper layers with a thickness of 25 $\mu \mathrm{m}$ and $9 \mu \mathrm{m}$, respectively. The PMS is integrated directly underneath the antenna to achieve a compact module and to prevent antenna radiation from coupling into the circuitry. Moreover, only small electronic components (maximum footprint is $3 \times 3 \mathrm{~mm}$ ) are used and these are distributed over a larger area than necessary to maintain the flexibility of the design. A non-conductive adhesive sheet is used to fix the solar cell to the slot plane. A similar sheet attaches the PMS and MEC to the feed plane without covering the feed line, as depicted in Fig. 1.c. Note that the implementation of the PMS depicted in Fig. 1.c. also contains additional test pins and push buttons for measurement purposes. Therefore, the dimensions of the PMS can be further reduced in the final application.

\section{Simulation AND MEASUREMENT RESUlts}

\section{A. Antenna performance}

First, the input matching of the antenna in free space was verified in an anechoic chamber by means of an Agilent N5242A PNA-X Network Analyzer. The measured and simulated reflection coefficients $\left|S_{11}\right|$, shown in Fig. 2, are in good agreement. Impedance matching over the entire ISM band and both uplink and downlink of the 4G LTE band 7 is obtained with a measured bandwidth of $409 \mathrm{MHz}$. The measured and simulated radiation patterns in the E- and $\mathrm{H}$ plane of the antenna at $2.45 \mathrm{GHz}$ are shown in Fig. 3. Similar radiation patterns are obtained at $2.53 \mathrm{GHz}$ and $2.65 \mathrm{GHz}$. Measured and simulated antenna gain along broadside, FTBR and $\eta_{\text {rad }}$ at $2.45 \mathrm{GHz}, 2.53 \mathrm{GHz}$ and $2.65 \mathrm{GHz}$ are described in Table II. Antenna radiation efficiencies were determined by measuring the $3 \mathrm{D}$ radiation patterns at each specific frequency. Compared to a similar antenna presented in [8], a significantly higher $\eta_{\text {rad }}$ is obtained with our design. Second, an on-body $\left|S_{11}\right|$ measurement was performed by deploying the antenna on the chest of a person wearing a T-shirt. The corresponding curve in Fig. 2 exhibits a slight increase in bandwidth due to some additional losses because of body absorption. Yet, the radiation patterns in Fig. 3.b, demonstrate a similar gain along broadside as in free-space conditions whereas proximity of the body results in lower backside radiation. Third, the $\left|S_{11}\right|$ and radiation patterns of the antenna with integrated solar harvesting system (SHS) were measured in free-space conditions. Figs. 2 and 3 prove that the integration of solar cell, PMS and MEC has a minor influence on antenna performance. Finally, the antenna was bent with a radius of $5 \mathrm{~cm}$ along $\mathrm{H}$ - and E-planes to simulate the antenna deployed on a human arm. Fig. 2 shows that the antenna remains matched over the frequency ranges of interest in both cases, demonstrating its robustness under extreme bending.

\section{B. Energy harvesting performance in a real life situation}

The solar cell's maximum power point was measured to be $78.6 \mathrm{~mW}$ using a solar simulator $\left(100 \mathrm{~mW} / \mathrm{cm}^{2}\right)$. This illumination level corresponds to sunlight directly overhead on a turbidity free sky. In a practical application the power output of the solar cells will be lower due to overcast and skew incidence of the solar rays. Therefore, following measurement setup was considered to characterize and validate our system in a real-life situation. A test person, wearing the proposed system on his chest, is standing outside in an upright position in such a manner that the flexible solar cell is perpendicular to the earth surface and pointing in the direction of the sun. An adjustable constant current source (a current mirror, copying a stable and accurate reference current set by a trimmer and the Fluke Philips PM2811) is connected as a load (sink) to the regulated voltage output of the PMS. Then the load current was swept from 1 to $15 \mathrm{~mA}$ and the power delivered by the solar cell to the PMS was measured. Each specific load current was applied over a sufficiently long time interval to yield continuous load conditions. The measurement started at 2 p.m. local time in Ghent (latitude: $51^{\circ} 2^{\prime} 46^{\prime}$ ', longitude: $\left.3^{\circ} 43^{\prime} 37^{\prime \prime}\right)$. The elevation angle of the sun was $51.45^{\circ}$ and the sky was turbidity free. The scavenged power as a function of the continuous load current was measured and is depicted in Fig. 4. In this specific real-life case, the maximum harvested DC output power decreased to $53 \mathrm{~mW}$, occurring at a continuous load current of $13 \mathrm{~mA}$. Moreover, the SHS is able to sustain continuous load currents up to $14 \mathrm{~mA}$. Furthermore, with the same measurement setup but with open-circuited regulated output and a fully discharged MEC (discharge current of $50 \mu \mathrm{A}$ ), it took 11 minutes and 30 seconds to charge the connected MEC to $50 \%$ of its nominal capacity. However, it took only 6 minutes to harvest the exact same amount of energy with two parallel connected initially fully discharged MECs. This can be attributed to a reduced total series resistance of the cells and a shifted operation point of the solar cell allowing an increased total charge current. 
These measurements demonstrate good performance of the fully-integrated SHS. Yet, depending on the application, an even better performance can be achieved by integrating more MECs and/or more flexible solar cells or tailoring the PMS to the power profile of the communication module.

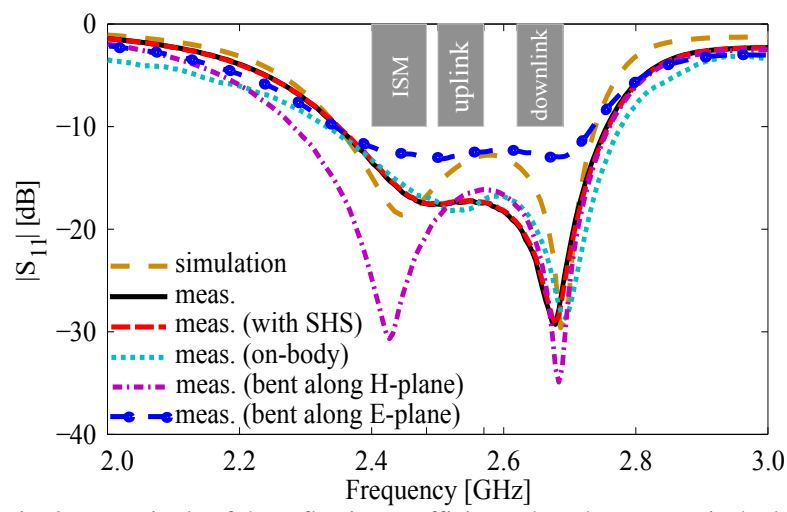

Fig. 2. Magnitude of the reflection coefficient when the antenna is deployed in different conditions.

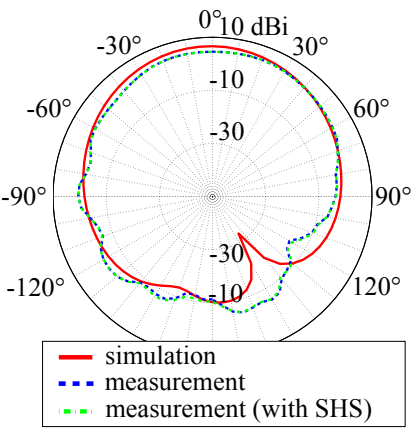

(a)

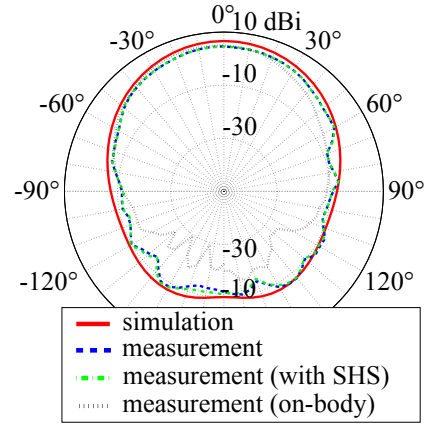
(b)
Fig. 3. Measured and simulated radiation pattern [dBi] of the antenna at 2.45 $\mathrm{GHz}$ in the E-plane (a) and H-plane (b).

TABLE II

MEASURED (SIMULATED) ANTENNA CHARACTERISTICS

\begin{tabular}{llll}
\hline \hline & Gain [dBi] & FTBR [dB] & $\eta_{\text {rad }}[\%]$ \\
\hline \hline $2.45 \mathrm{GHz}$ & $4.7(6.6)$ & $17.1(16.7)$ & $89(83)$ \\
$2.53 \mathrm{GHz}$ & $4.9(6.5)$ & $17.9(15.1)$ & $93(83)$ \\
$2.65 \mathrm{GHz}$ & $5.1(6.1)$ & $16.3(13.6)$ & $92(75)$ \\
\hline \hline
\end{tabular}

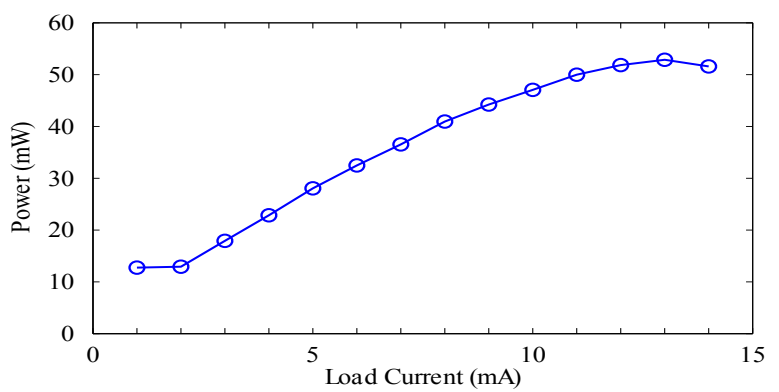

Fig. 4. Power delivered by the solar cell to the PMS as a function of load current of the regulated output in the described real-life situation.

\section{CONCLUSION}

A novel dual-band wearable textile antenna based on substrate integrated waveguide (SIW) technology for operation in the [2.4-2.4835] GHz ISM band and the [2.5$2.69 \mathrm{GHz}]$ 4G LTE band 7 was designed and validated. The design exhibits large radiation efficiency, high front-to-back ratio and large bandwidth. Its high robustness against bending, low influence of human body and compact flexible structure make the proposed antenna extremely suitable for wearable applications. The surface required by the antenna is reused for integration of a solar energy harvesting and energy management system. This novel integration of both energy scavenging and power management hardware onto a wearable textile antenna allows a more compact overall SFIT system and improves user comfort.

\section{REFERENCES}

P. Vanveerdeghem, B. Jooris, P. Becue, P. Van Torre, H. Rogier, I. Moerman, and J. Knockaert, "Reducing Power Consumption in Body-centric Zigbee Communication Links by means of Wearable Textile Antennas," in 2nd International Workshop on Measurement-based Experimental Research, Methodology and Tools (MERMAT), Dublin, Ireland, May 7, 2013.

A. Dierck, H. Rogier, and F. Declercq, "A Wearable Active Antenna for Global Positioning System and Satellite Phone," IEEE Trans. Antennas Propag., vol. 61, no. 2, pp. 532-538, Feb. 2013. V. Leonov, T. Torfs, C. Van Hoof, and R. J. M. Vullers, "Smart Wireless Sensors Integrated in Clothing: an Electrocardiography System in a Shirt Powered Using Human Body Heat," Sens. \& Transd. J., vol. 107, no. 8, pp. 165-176, 2009.

G. Orecchini, L. Yang, M. Tentzeris, and L. Roselli, "Smart Shoe: An autonomous inkjet-printed RFID system scavenging walking energy," in Proc. 2011 IEEE Int. Symp., Antennas and Propagation, Spokane, WA, USA, Jul. 3-8, 2011, pp. 1417-1420. F. Declercq, A. Georgiadis, and H. Rogier, "Wearable ApertureCoupled Shorted Solar Patch Antenna for Remote Tracking and Monitoring Applications," in Proc. of the 5th European Conference on Antennas and Propagation (EUCAP), Rome, Italy, Apr. 11-15, 2011, pp. 2992-2996.

A. Collado, and A. Georgiadis, "Conformal Hybrid Solar and Electromagnetic (EM) Energy Harvesting Rectenna," IEEE Trans. Circuits Syst. I, Regul. Pap., vol. 60, no. 8, pp. 2225-2234, Aug. 2013.

M. Mantash, S. Collardey, A.-C. Tarot, and A. Presse, "Dual-band WiFi and 4G LTE Textile Antenna," in 7th European Conference on Antennas and Propagation (EuCAP), Gothenburg, Sweden, Apr. 8-12, 2013, pp. 422-425.

R. Moro, S. Agneessens, H. Rogier, and M. Bozzi, "Wearable Textile Antenna in Substrate Integrated Waveguide Technology," IET Electron. Lett., vol. 48, no. 16, pp. 985-987, Aug. 2012.

T. Kaufmann, and C. Fumeaux, "Wearable Textile Half-Mode Substrate-Integrated Cavity Antenna Using Embroidered Vias," IEEE AntennasWirel. Propag. Lett. , vol. 12, pp. 805-808, 2013.

G. Q. Luo, Z. F. Hu, L. X. Dong, and L. L. Sun, "Planar slot antenna backed by substrate integrated waveguide cavity," IEEE Antennas Wireless Propag. Lett., vol. 7, no. 1, pp. 236-239, 2008. K. Gong, Z. Chen, X. Qing, P. Chen, and W. Hong, "Substrate Integrated Waveguide Cavity-Backed Wide Slot Antenna for 60GHz Bands," IEEE Trans. Antennas Propag., vol. 60, no. 12, pp. 6023-6026, 2012.

W. Yang, and J. Zhou, "Wideband Low-Profile Substrate Integrated Waveguide Cavity-Backed E-shaped Patch Antenna," IEEE Antennas Wireless Propag. Lett., vol. 12, pp. 143-146, 2013. G. Q. Luo, Z. F. Hu, W. J. Li, X. H. Zhang, L. L. Sun, and J. F. Zheng, "Bandwidth-Enhanced Low-Profile Cavity-Backed Slot Antenna by Using Hybrid SIW Cavity Modes," IEEE Trans. Antennas Propag., vol. 60, no. 4, pp. 1698-1704, 2012.

F. Declercq, I. Couckuyt, H. Rogier, and T. Dhaene, "Environmental High Frequency Characterization of Fabrics Based on a Novel Surrogate Modelling Antenna Technique," IEEE Trans. Antennas Propag., vol. 61, no. 10, pp. 5200-5213, Oct. 2013.

Maxim Integrated. "MAX17710: Energy-Harvesting Charger and Protector," Accessed on 27 June 2013; Available online: http://datasheets.maximintegrated.com/en/ds/MAX17710.pdf. 